Распространенность метаболического синдрома (MC) имеет мировую тенденцию к увеличению и зависит от многих составляющих, что объясняет сложности диагностики и подходов к профилактике и лечению данной патологии. Возраст, образ жизни, социально-экономический статус, инсулинорезистентность (ИР), дислипидемия, ожирение и генетическая предрасположенность являются факторами, влияющими на риск развития и прогрессирования МС. Особенности распределения жировой ткани и ее дисфункция - важные факторы развития ИР с ожирением, а также риска формирования кардиометаболических заболеваний и МС. Понимание данных механизмов связывают с достижениями в области метаболического фенотипирования. Метаболическое фенотипирование лиц, страдающих ожирением, важно в отношении изучения патофизиологии нарушений обмена веществ, возможного сопутствующего заболевания и поиска инновационных стратегий профилактики и лечения МС. Поэтому актуальность дальнейшего изучения патофизиологических механизмов, лежащих в основе различных метаболических фенотипов МС, является одним из перспективных направлений современных научных исследований. В настоящем обзоре суммированы современные литературные данные о распространенности МС в зависимости от пола, возраста, популяции, территории проживания, образования, уровня физической активности и многих других параметров. Детализированы метаболические риски развития МС. Рассмотрены биологические маркеры МС. Показана необходимость метаболического фенотипирования МС, которое может иметь потенциальное терапевтическое значение.

КЛЮЧЕВЫЕ СЛОВА: метаболический синдром; распространенность; метаболическое фенотипирование.

\title{
PREVALENCE AND BIOMARKERS IN METABOLIC SYNDROME
}

\section{(c) Oxana Yu. Kytikova*, Marina V. Antonyuk1', Tatyana A. Kantur², Tatyana P. Novgorodtseva' , Yulia K. Denisenko}

${ }^{1}$ Far Eastern Scientific Centre of Physiology and Pathology of Respiration, Vladivostok, Russia.

${ }^{2}$ Far Eastern Federal University, Vladivostok, Russia

The prevalence of metabolic syndrome (MS) has a worldwide tendency to increase and depends on many components, which explains the complexity of diagnostics and approaches to the prevention and treatment of this pathology. Age, lifestyle, socioeconomic status, insulin resistance (IR), dyslipidemia, obesity and genetic predisposition are factors influencing the risk of developing and progression of MS. Features of the distribution and dysfunction of adipose tissue are important factors in the development of IR, with obesity, as well as the risk of the formation of cardiometabolic diseases and MS. Understanding of mechanisms is linked to advances in metabolic phenotyping. Metabolic phenotyping of obese persons is important for the development of important diseases in relation to the study of the pathophysiology of metabolic disorders, the possible concomitant disease and the search for innovative strategies for the prevention and treatment of MS. The understanding of MS mechanisms is associated with advances in metabolic phenotyping. Therefore, the relevance of further study of the pathophysiological mechanisms underlying various metabolic phenotypes of MS is one of the promising areas of modern scientific research. This review summarizes the current literature data on the prevalence of MS depending on gender, age, population, area of residence, education, level of physical activity, and many other parameters. Metabolic risks of MS development are detailed. Biological markers of MS are considered. The necessity of metabolic phenotyping of MS has been shown, which may have potential therapeutic value.

KEYWORDS: metabolic syndrome; prevalence; metabolic phenotyping.

\section{ВВЕДЕНИЕ}

Метаболический синдром (МС) определяется как группа метаболических нарушений, представленных рядом факторов риска развития сердечно-сосудистых патологий и сахарного диабета 2 типа (СД2) [1]. Связанными с МС компонентами являются отложение абдоминального жира, артериальная гипертензия, нарушения углеводного обмена и дислипидемия [2]. Взаимосвязь между МС и развитием многих хронических заболеваний (сердечно-сосудистая патология, неалкогольная жировая болезнь печени (НАЖБП), артриты, хронические заболевания почек, шизофрения), а также некоторых видов рака (рак эндометрия, простаты, колоректальный рак и рак молочной железы) отмечается на протяжении многих десятилетий [3-6]. Известно, что МС также развивается параллельно с ожирением и СД2 [7]. 
Ожирение является распространенным во всем мире нарушением метаболизма, приобретающим характер мировой пандемии. В 2015 г. ожирением страдали 604 млн взрослых и 108 млн детей. С 1980 г. распространенность ожирения удвоилась в 73 странах мира [8]. Согласно данным Организации Объединенных Наций $(\mathrm{OOH})$, по количеству больных ожирением первое место в мире занимает Мексика (32,8\%), РФ находится на 19-м месте (24,9\%). С глобальным ростом ожирения МС стал одной из основных проблем общественного здравоохранения во всем мире [9-11].

Распространенность и смертность от диабета также продолжают расти во всем мире, что имеет важные медико-социальные последствия для системы здравоохранения. В исследовании K. Ogurtsova и соавт. было проанализировано 196 источников данных из 111 стран [12]. По оценкам, в 2015 г. насчитывалось 415 млн людей с диабетом в возрасте 20-79 лет, 5 млн смертей, связанных с диабетом, а общие глобальные расходы системы здравоохранения оценивались в 673 млрд долларов США. Около 75\% людей с диабетом проживали в странах с низким и средним уровнем дохода. Прогнозируется, что число людей с диабетом в возрасте 20-79 лет увеличится до 642 млн к 2040 г. [12].

Аналогичных глобальных данных о распространенности МС нет, но поскольку он встречается примерно в три раза чаще, чем диабет, предполагаемая распространенность данного симптомокомплекса составляет около четверти населения мира [11]. Около 1/5 населения стран Азиатско-Тихоокеанского региона, в котором проживает половина популяции мира, имеет MC [13]. Иными словами, в настоящее время более 1 млрд человек в мире страдают MC, и заболеваемость данной патологией будет неуклонно расти.
В базе данных PubMed проводился поиск первичной информации (полнотекстовые и реферативные базы данных) по выбранным критериям включения. Информационные запросы включали следующую совокупность ключевых слов: «метаболический синдром; распространенность; метаболическое фенотипирование» и период публикаций «2010-2021». В результате проведенного анализа идентифицированных статей нами установлено, что только небольшое их количество является обзорными работами, что указывает на необходимость систематизации накопленных в литературе данных. В настоящем обзоре суммированы современные литературные данные о распространенности МС в зависимости от пола, возраста, популяции, территории проживания, образования, уровня физической активности и многих других параметров. Детализированы метаболические риски развития МС. Рассмотрены биологические маркеры МС. Показана необходимость метаболического фенотипирования МС, которое может иметь потенциальное терапевтическое значение.

Критерии метаболического синдрома

Показатели распространенности МС варьируют в зависимости от критериев, используемых для его определения [13]. Известно, что для диагностики МС применяются критерии нескольких медицинских сообществ: WHO (World Health Organization), NCEPATP III (National Cholesterol Education Program-Adult Treatment Panel III), AACE (American Association of Clinical Endocrinologists), IDF (International Diabetes Federation), EGIR (European Group for the Study of Insulin Resistance), Международного института метаболического синдрома и Всероссийского научного общества кардиологов (ВНОК) [1, 10, 11, 14-17]. Определения МС, выдвинутые перечисленными сообществами, существенно отличались критериями, степенью их важности, а также интервалами включенных параметров (табл. 1).

Таблица 1. Сравнительная характеристика критериев метаболического синдрома, выдвигаемых некоторыми медицинскими сообществами (WHO, EGIR, NCEP ATP III, AACE)

\begin{tabular}{|c|c|c|c|c|}
\hline \multicolumn{5}{|c|}{ Критерии диагностики МС } \\
\hline & $\begin{array}{c}\text { WHO } \\
{[10]}\end{array}$ & $\begin{array}{l}\text { EGIR } \\
{[11]}\end{array}$ & $\begin{array}{l}\text { NCEP ATP III } \\
{[16]}\end{array}$ & $\begin{array}{l}\text { AACE } \\
{[17]}\end{array}$ \\
\hline ИР/ГИ & $\begin{array}{l}\text { ИР - верхний } 25 \% \\
\text { квартиль для N, } \\
\text { и/или гипергликемия } \\
\text { натощак, и/или } \\
\text { нарушенная толерантность } \\
\text { к глюкозе, и/или СД 2-го типа }\end{array}$ & $\begin{array}{l}\text { ГИ - верхний } \\
25 \% \text { квартиль } \\
\text { для N }\end{array}$ & - & $\begin{array}{l}\text { Высокий риск } \\
\text { ОТ } \geq 102 \text { (муж.) } \\
\text { ОТ } \geq 88 \text { (жен.) } \\
\text { или } \\
\text { ИМТ } \geq 25\end{array}$ \\
\hline Число признаков & +2 любых & +2 любых & Любые 3 & +2 любых \\
\hline Глюкоза, ммоль/л (мг/дл) & - & $\geq 6,1(110)$ & $\geq 6,1(110)$ & $\geq 6,1(110)$ \\
\hline $\begin{array}{l}\text { Липопротеиды высокой } \\
\text { плотности, ммоль/л (мг/дл) }\end{array}$ & $\begin{array}{l}\leq 0,9 \text { (35) (муж.) } \\
<1,0 \text { (40) (жен.) }\end{array}$ & $<1,0(40)$ & $\begin{array}{l}<1,0 \text { (40) (муж.) } \\
<1,3 \text { (50) (жен.) }\end{array}$ & $\begin{array}{l}<1,0 \text { (40) (муж.) } \\
<1,3 \text { (50) (жен.) }\end{array}$ \\
\hline Триглицериды, ммоль/л (мг/дл) & $\geq 1,7(150)$ & $\geq 2,0(180)$ & $\geq 1,79(150)$ & $\geq 1,7(150)$ \\
\hline Ожирение & $\begin{array}{l}\text { ОТ/ОБ>0,9 (муж.) } \\
\text { ОТ/ОБ>0,85 (жен.) } \\
\text { ИМТ } \geq 30\end{array}$ & $\begin{array}{l}\text { ОТ } \geq 94 \text { (муж.) } \\
\text { ОТ } \geq 80 \text { (жен.) }\end{array}$ & $\begin{array}{l}\mathrm{OT} \geq 102 \text { (муж.) } \\
\mathrm{OT} \geq 88 \text { (жен.) }\end{array}$ & \\
\hline
\end{tabular}

АД, мм рт.ст.

$\geq 140$ (90)

$\geq 140(90)$

$\geq 130$ (85)

$\geq 130$ (85)

Примечание. АД - артериальное давление; ГИ — гиперинсулинемия; ИР — инсулинорезистентность; ИМТ — индекс массы тела; ОТ — окружность талии; ОБ - окружность бедер. 
Таблица 2. Унифицированные критерии метаболического синдрома (IDF, NHLBI, AHA, WHF, IAS, IASO, 2009 г.) [15]

\section{Критерии метаболического синдрома}

1. Абдоминальное ожирение (данные, специфичные для отдельных стран и популяций).

2. Триглицериды >1,7 ммоль/л (150 мг/дл) или медикаментозная терапия гипертриглицеридемии.

Любые три из данных критериев
3. Снижение уровня липопротеидов высокой плотности: у мужчин: <1,0 ммоль/л (40 мг/дл), у женщин: <1,3 ммоль/л (50 мг/дл) или медикаментозная терапия дислипидемии.

4. Повышенный уровень АД, 130/85 мм рт. ст., или прием антигипертензивной терапии пациентом с артериальной гипертензией в анамнезе.

5. Повышенный уровень гликемии натощак, 100 мг/дл, или прием сахароснижающих препаратов

Таблица 3. Критерии метаболического синдрома (ВНОК, 2013 г.) [1]

\section{Критерии метаболического синдрома}

\begin{tabular}{ll}
\hline Основной критерий & Центральное ожирение: ОТ муж. $>94$ см, жен. $>80$ см \\
\hline & 1.АД $\geq 140 / 90$ мм рт. ст. \\
& 2. Липопротеиды высокой плотности $<1,2$ ммоль/л (жен.) и <1,0 ммоль/л (муж.). \\
& 3. Триглицериды $\geq 1,7$ ммоль/л. \\
& 4. Липопротеиды низкой плотности $>3,0$ ммоль/л. \\
& 5. Нарушенная толерантность к глюкозе - повышенный уровень глюкозы плазмы \\
& через 2 ч после нагрузки 75 г безводной глюкозы при применении перорального \\
глюкозотолерантного теста $\geq 7,8$ и <11,1 ммоль/л, при условии, что уровень глюкозы \\
Два дополнительных \\
притерия
\end{tabular}

6. Нарушенная гликемия натощак - повышенный уровень глюкозы плазмы натощак $\geq 6,1$ и <7,0 ммоль/л, при условии, что глюкоза плазмы через 2 ч при применении перорального глюкозотолерантного теста <7,8 ммоль/л.

7. Комбинированное нарушение. Нарушенная гликемия натощак/Нарушенная толерантность к глюкозе - повышенный уровень глюкозы плазмы натощак $\geq 6,1$ и $<7,0$ ммоль/л в сочетании с глюкозой плазмы через 2 ч при применении перорального глюкозотолерантного теста $\geq 7,8$ и < 11,1 ммоль/л
В 2009 г. для унификации критериев диагностики МС было принято заключение Международной федерации диабета (IDF), Национального института сердца, легких и крови (NHLBI), Американской кардиологической ассоциации (AHA), Всемирной кардиологической федерации (WHF), Международного общества атеросклероза (IAS) и Международной ассоциации по изучению ожирения (IASO). Coгласно данному заключению, для постановки диагноза МC необходимо наличие трех из пяти перечисленных критериев. Для диагностики абдоминального ожирения рекомендовано пользоваться пороговыми значениями ОТ, специфичными для отдельных стран и популяций [15] (табл. 2).

В России первые рекомендации по диагностике и лечению МС были утверждены в 2007 г. на конгрессе Всероссийского научного общества кардиологов (ВНОК). Национальные рекомендации 2013 г. (ВНОК, 2013) основываются на критериях, перечисленных в таблице $3[1,14]$.

Разнообразие диагностических критериев МС свидетельствует о том, что МС является с одной стороны чрезвычайно гетерогенным патологическим состоянием, в развитии которого могут принимать участие многие факторы, с другой - затрудняет анализ распространен- ности МС в различных популяциях. В этой связи, изучение эпидемиологических аспектов МС является одним из актуальных направлений современных исследований.

\section{ФАКТОРЫ, ОБУСЛОВЛИВАЮЩИЕ РАСПРОСТРАНЕННОСТЬ МЕТАБОЛИЧЕСКОГО СИНДРОМА}

Стоит учитывать, что распространенность МС зависит от пола, возраста, этнического происхождения, территории проживания, образования, уровня физической активности и многих других параметров и факторов.

Так, распространенность МС наиболее низкая в Китае (15,2-21,1\% населения) [18] и на Филиппинах (11,9\%) [13], в то время как среди населения США данный показатель составляет 34,2\% [19]. В Российской Федерации (РФ) МС встречается в диапазоне 40,3\% - 50,5\% [1]. Высокая распространенность данной патологии также характерна для Пакистана (49,0\%) [13]. Стоит отметить, что по последним данным, заболеваемость МС имеет тенденцию к увеличению в Китае, Южной Kорее и Тайване [13]. Эпидемический рост заболеваемости МС 
в течение короткого периода времени указывает на второстепенную роль генетической предрасположенности в этиопатогенезе данного заболевания. Кроме того, дети, рожденные от матери или отца с ожирением, которые перенесли бариатрическую операцию до зачатия детей, менее подвержены ожирению и развитию МС, чем дети, рожденные до бариатрической операции [20]. Тем не менее эпигенетика все же играет значимую роль в развитии MC, особенно в азиатских и европейских когортах [21]. Множественные генетические локусы, связанные с МС и его компонентами, были идентифицированы в рамках исследований по геномной ассоциации (GWAS) [22]. Подтвержден 21 локус, из которых 4 были описаны только недавно: rs765547 рядом с LPL; rs3782889 в MYL2; rs11065756, rs10849915 в ССDC63. Исследования азиатских и европейских когорт населения показали высокую степень наследуемости [23]. Наследуемость МС в Kopeе составляет 50-60 \% [23]. В то же время вариант гена rs9939609, связанный с массой жира и ожирением, коррелирует с развитием МС преимущественно у европейцев [21]. Таким образом, генетическая предрасположенность является значимой составляющей патогенеза MC и может также влиять на его распространенность в азиатских и европейских когортах.

Неясно, в какой степени компоненты МС различаются среди населения с разным этническим происхождением. Например, превалирующими компонентами МС у индонезийцев были гипертония (61\%) и гипергликемия (51\%), у населения Нидерландов - гипертония (62\%) и абдоминальное ожирение (40\%) [24]. Американцы из Южной Азии имели высокую частоту встречаемости абдоминального ожирения [25]. Более половины больных диабетом живут в Юго-Восточной Азии и Западной части Тихого океана. В ближайшие 25 лет ожидается, что рост заболеваемости диабетом будет особенно значительным в Африке [12]. Заболеваемость диабетом выше у коренных народов США (15\% среди американских индейцев), но ниже среди китайцев - 4,3\%.

Интересны гендерные различия в распространенности МС. В большинстве исследований сообщалось о более Высокой распространенности МС у женщин $[6,13]$. Так, распространенность МС в Китае составила 17,1-19,6\% среди мужчин и 13,0\%-22,7\% среди женщин [18]. У жительниц Индонезии данный показатель превышает заболеваемость МС среди лиц мужского пола (46\% и 28\% соответственно) [24]. Однако распространенность МС в высокогорных районах Китая выше у мужчин (5,9\%) в сравнении с женщинами (1,8\%) [26]. У мужчин, проживающих в Нидерландах, также заболеваемость МС выше (36\%), чем у женщин (24\%) [24].

В большинстве исследований сообщалось о более высокой распространенности МС у жителей городов [13]. В то же время в Китае МС чаще встречается у сельских жителей мужского пола, в то время как у взрослых городских жителей более распространены гипертония и ожирение. Так, распространенность МС в городе и сельской местности составила соответственно 6,6\% (11,9\% у мужчин и 1,5\% у женщин) против 3,0\% (4,6\% у мужчин и 1,8\% у женщин) [26]. Текущие данные другого исследования указывают на высокую распространенность МС среди сельских жителей Китая, при этом чаще данное заболевание встречается у женщин [6].
Во всех эпидемиологических исследованиях распространенность МС увеличивается с возрастом, что обусловлено общностью биохимических изменений данного симптомокомплекса и старения [10, 27]. Результаты исследований N. Ivanova и соавт. позволяют предположить, что нейровоспаление белого вещества головного мозга, сопровождающее старение, может быть одним из возможных процессов, лежащих в основе развития МС и болезни Альцгеймера [27]. Присутствие только одного компонента МС существенно увеличивает риск его развития в более позднем возрасте [28]. Показано, что высокая распространенность МС также наблюдается среди пожилых людей с низким уровнем физической активности [29]. Наличие МС среди лиц старшего возраста чаще коррелирует с дислипидемией [29]. Распространенность данной патологии увеличивается при старении у лиц обоих полов. В то же время, несмотря на то, что симптомы депрессии, наблюдаемые в пожилом возрасте, были связаны с компонентами МС (повышенным уровнем глюкозы в плазме натощак и диабетом), эта связь была менее выражена у женщин [30]. Нарушения сна также характерны для лиц старшей возрастной группы. Как короткий (<6 ч), так и длительный сон (>9 4) связан с повышенным риском развития МС [31]. Продолжительность сна может быть определяющим фактором метаболического здоровья у пожилых, так как сон играет важную роль в поддержании гомеостаза внутренней среды, которая может регулировать физиологические, гормональные и психологические процессы [32].

Интересно, что МС присутствует у 4,8-7\% молодых людей (до 30 лет) [28]. Атерогенная дислипидемия, определяемая как изменение уровня липопротеинов низкой плотности (ЛПНП), была наиболее распространенным компонентом МС у молодых людей $(26,9-41,2 \%)$, за ним следовали повышение артериального давления (16,6-26,6\%), абдоминальное ожирение (6,8-23,6\%), атерогенная дислипидемия, определяемая как повышение уровня триглицеридов (8,6-15,6\%) и повышение уровня глюкозы натощак $(2,8-15,4 \%)$.

Другими факторами, независимо связанными с распространенностью МС, являются низкий уровень образования, физической активности и состояние менопаузы $[6,18,29]$. Физическая активность средней интенсивности может снизить риск развития МС, диабета 2 типа, СС3 и инсульта [33]. Комплексная программа сердечно-сосудистой реабилитации и изменения образа жизни является эффективным подходом к лечению пациентов с MC, оказывая положительное краткосрочное и долгосрочное влияние на контроль веса, уровень холестерина и триглицеридов [9]. Начало менопаузы имеет тенденцию увеличивать частоту общего и абдоминального ожирения [34] и риск развития МС [18]. Различия в степени выраженности абдоминального ожирения также ответственны за этнические различия в распространенности МС. Хорошо известно, что избыток висцеральной жировой ткани тесно связан с кардиометаболическими осложнениями и поэтому может объяснить и кардиометаболические различия, наблюдаемые между этническими группами [35].

Таким образом, распространенность МС имеет тенденцию к увеличению и зависит от многих составляющих, что объясняет сложности диагностики и подходов 
к профилактике и лечению данной патологии. Одним из основных критериев, которые необходимо учитывать при разработке методов лечения и профилактики данной патологии, является метаболическое здоровье [36].

\section{МЕТАБОЛИЧЕСКОЕ ФЕНОТИПИРОВАНИЕ}

Ожирение является основным компонентом МС и рассматривается как центральное патогенетическое звено синдромокомплекса [14]. Известно, что развитие ожирения сопровождается накоплением жировой ткани не только в местах ее физиологической локализации, но и в других органах [37]. Среди лиц с признаками избыточной массы тела и ожирения выделяют несколько подгрупп с разными метаболическими фенотипами: три метаболически здоровых фенотипа (с нормальной массой тела, с избыточной массой тела, с ожирением) и три метаболически нездоровых фенотипа (с нормальной массой тела, с избыточной массой тела, с ожирением). Phillips C.M. детально описывает используемые в настоящее время критерии для определения метаболически здорового фенотипа среди детей, подростков и взрослых [38]. Хотя метаболическое здоровье можно определить как отсутствие инсулинорезистентности, текущее определение метаболического здоровья основано на отсутствии MC (или некоторых из его кардиометаболических показателей - дислипидемии, нарушенной резистентности к инсулину, артериальной гипертензии) у людей с избыточной массой тела. Дополнительным критерием является благоприятный воспалительный статус, определяемый уровнем С-реактивного белка [38].

В ряде исследований продемонстрировано, что около 10-30\% лиц, страдающих ожирением, не имеют признаков МС и считаются метаболически здоровыми [37]. По другим данным, истинное метаболически здоровое ожирение представляет собой редкое явление и встречается в диапазоне от 3 до 6\% среди пациентов, имеющих индекс массы тела (ИМТ) >30 кг/м² [39]. Очевидно, что абсолютное количество жира в организме не полностью отражает метаболическое здоровье и не может объяснить повышенный метаболический риск развития нарушений обмена веществ, развития резистентности к инсулину, МС, СД2 и сердечно-сосудистых заболеваний (СС3) у пациентов, страдающих ожирением [36]. Существует ряд антропометрических показателей, характеризующих количество жировой ткани, к которым относятся ИМТ, объем талии (ОТ), соотношение ОТ, роста и объема бедер (ОБ), сагиттальный абдоминальный диаметр, индекс висцерального ожирения (The Visceral Adiposity Index (VAI)), индекс стеатоза печени (Fatty Liver Index (FLI)) и "body adiposity index (BAI)". Для диагностики ожирения важна оценка его абдоминального типа посредством измерения ОТ и определения количества общей жировой массы в организме, поскольку ее повышенное процентное содержание может быть и у лиц с нормальными значениями ИМТ. Абдоминальное накопление жира относится к метаболически нездоровому фенотипу ожирения и связано с нарушением обмена веществ, развитием резистентности к инсулину и с высоким риском развития МС, СС3, СД2, тогда как общее ожирение - нет [34]. Например, один из фенотипов МС (the hypertriglyceridemic-waist (HW) phenotype) характе- ризуется одновременным увеличением окружности талии и концентрацией триглицеридов, которые являются важными предикторами хронических заболеваний и патологических состояний, таких как ишемическая болезнь сердца и нарушение метаболизма глюкозы. В основе развития метаболически нездорового ожирения лежит не избыточное накопление жировой ткани, а изменения в функционировании адипоцитов и распределении жировой ткани в организме [37]. Такой вид ожирения характеризуется уменьшением подкожно-жировой клетчатки, гипертрофией висцеральных адипоцитов, провоспалительным состояние жировой ткани.

Значение каждого фенотипа в развитии ассоциированных с ожирением заболеваний (СС3, СД2, НАЖБП) обсуждается. Например, в исследовании N. Eckel и coавт. установлено, что лица с метаболически здоровым ожирением имеют повышенный риск развития СС3 в сравнении с метаболически здоровым фенотипом с нормальной массой тела. Однако этот риск был ниже, чем у лиц с метаболически нездоровым фенотипом ожирения и лиц с метаболически нездоровым фенотипом и нормальной массой тела. Кроме того, в группе с метаболически здоровым ожирением авторы наблюдали более высокий риск среди лиц мужского пола в сравнении с женским [40]. Метаболически здоровое ожирение связано с более высоким риском развития НАЖБП, независимо от висцерального ожирения [41]. Таким образом, лица, страдающие метаболически здоровым ожирением, также имеют риск развития заболеваний, ассоциированных с ожирением, который выше, чем у лиц с нормальным ИМТ. Метаболически здоровое ожирение является показанием к коррекции массы тела и модификации факторов риска.

Известно, что нарушение липидного обмена способствует развитию ожирения, диабета и $\mathrm{MC}[42,43]$. Липидные изменения могут быть как причиной, так и следствием нарушения метаболизма глюкозы, в результате которого развивается резистентность к инсулину. Соответственно, МС и другие метаболические заболевания и состояния (гипергликемия, дислипидемия, гипертония и сахарный диабет 2 типа), характеризующиеся нарушениями в метаболизме глюкозы или липидов, тесно взаимосвязаны [44]. Однако общая точка зрения на взаимоотношения между МС и другими метаболическими заболеваниями отсутствует $[42,45]$. Обычная диагностика и лечение этих заболеваний упускает из виду их взаимосвязь и гетерогенность. В работе Di Chen и соавт. было показано, что данные заболевания можно классифицировать на определенные метаболические подтипы, базирующиеся на показателях липидного обмена, метаболизма глюкозы, аминокислот и иммунной системы [45]. Эти подгруппы могут представлять собой альтернативный способ классификации болезней обмена веществ и потенциального развития метаболических заболеваний, улучшая таким образом их лечение и профилактику.

Таким образом, метаболическое фенотипирование лиц, страдающих ожирением, является крайне важным в отношении изучения патофизиологии нарушений обмена веществ и выявления лиц высокого риска, что направлено на оптимизацию стратегии профилактики и лечения МС. Метаболомика признана мощным инструментом фенотипирования при хронических заболеваниях обмена 
веществ и может быть стратегией выбора для исследования МС и его компонентов $[46,47]$. Несмотря на то что в настоящее время внимание научно-исследовательских коллективов приковано к идентификации биомаркеров MC, их изучение с использованием метаболомики и липидомики является относительно новым направлением. Кроме того, метаболомика нацелена не только на идентификацию биомаркеров, но и на обнаружение эндогенных метаболитов, способных изменять фенотип клетки или организма. Метаболиты вовлечены в модуляцию различных процессов, таких как дифференцировка стволовых клеток, передача сигналов инсулина, иммунные ответы макрофагов и ряд других. Они играют разнообразную и важную роль в качестве сигнальных молекул, иммуномодуляторов и сенсоров окружающей среды. Это открывает возможность идентификации и применения метаболитов для воздействия на определенные фенотипы заболевания. В отличие от генов или белков, метаболиты легко доступны для тестирования и клинического использования.

\section{БИОМАРКЕРЫ МЕТАБОЛИЧЕСКОГО СИНДРОМА}

Метаболомика является полезным инструментом для выяснения сложности метаболизма и индивидуальных вариаций МС в целях развития стратифицированного подхода. В рамках исследования I. Surowiec и соавт. были определены несколько метаболитов, которые связаны с MC [46]. Известно, что индексы VAI и FLI являются ранними предикторами МС. В ряде исследований установлено, что с VAI и FLI положительно связан уровень метаболита кишечных бактерий триметиламин-N-оксида (trimethylamine-N-oxide (TMAO)) [48, 49]. В исследовании Barrea Luigi и соавт. установлено, что уровни ТМАО также увеличивались вместе с ИМТ. Эти результаты позволили авторам выдвинуть гипотезу о роли ТМАО как раннего биомаркера развития дисфункции жировой ткани и НАЖБП до развития МС [49]. Известно, что НАЖБП встречается в $100 \%$ случаев у больных с МС и его ведущими механизмами развития являются активация процессов липолиза, ИР и окислительный стресс (ОС) [5]. B работе X. Gaо и соавт. была изучена взаимосвязь между уровнем TMAO, L-карнитином сыворотки крови и компонентами MC (ожирение, артериальное давление, сывороточные липиды, уровень глюкозы в сыворотке крови и индекс резистентности к инсулину) [48]. Показано, что уровень L-карнитина был связан с нормальным уровнем глюкозы у больных MC, тогда как уровень ТМАО - с гипергликемией. Важность кишечного микробиома в развитии МС была подтверждена и в исследовании H. Plovier и соавт. [50]. Так, введение бактерий A. muciniphila корректировало параметры МС в экспериментальных условиях [50]. Примечательно, что полиметоксифлавоны цитрусовых положительно влияют на прогрессирование MC у мышей, которые получают высокожировую диету, за счет ингибирования дисбактериоза кишечника и регулирования метаболизма аминокислот [51].

Для СД2 характерна метаболическая картина, связанная в основном с измененным метаболизмом аминокислот, углеводов и микробиоты. При сочетании СД2 и МС наблюдался более низкий уровень углеводного 1,5-ангидроглюцитола [52]. Высокий уровень мочевой кислоты в сыворотке крови связан с повышенным риском развития СД2, гипертонией и МС [46]. Интересно, что некоторые из описанных биохимических путей развития МС связаны с ОС. Так, высокие уровни многих аминокислот (например, валина) при МС были связаны с имеющимся ОС [53].

Ацилкарнитины - класс соединений, который позволил по-новому взглянуть на биохимические пути, связанные с MC [46]. Ацилкарнитины необходимы для транспорта жирных кислот через мембрану митохондрий для $\beta$-окисления. Ацилкарнитины положительно коррелируют с уровнями глюкозы натощак [46]. Более высокие концентрации ацилкарнитинов в крови были связаны с ожирением, ИР и СД2. В экспериментальном исследовании in vitro M. Aichler и соавт. продемонстрировали, что высокие концентрации ацилкарнитина вызывают дисбаланс между синтезом и секрецией инсулина, что способствует развитию дисфункции бета-клеток в ткани поджелудочной железы [54]. Данный класс соединений также перспективен для изучения в качестве потенциального биомаркера МС.

Кетокислоты (альфа-кетоглутаровая, молочная и пировиноградная кислота) также коррелируют с МС. Важно отметить, что высокие уровни лактата как продукта окисления пировиноградной кислоты свидетельствуют об ОС. Триглицериды прямо коррелируют с МС, в то время как триацилглицерол имеет обратную корреляцию с несколькими компонентами синдрома [46]. Дальнейшие исследования могут помочь в изучении биомаркерной роли кетокислот при МС.

Фосфатидилхолин значительно повышен у пациентов с МС и имеет положительную корреляцию с окружностью талии, уровнем глюкозы в плазме, свободными жирными кислотами и уровнями триглицеридов, а также с провоспалительными маркерами, такими как интерлейкины (IL-1b и IL-8) [55]. Кроме того, обнаружена прямая корреляция фосфатидилхолина с лептином и отрицательная - с адипонектином. Вероятно, фосфатидилхолин может стать новым биомаркером MC, который указывает на развитие воспаления [55].

Жирные кислоты и их производные также рассматриваются в качестве значимых маркеров развития МС [43]. Novgorodtseva T.P. и соавт. был изучен липидом крови у пациентов с МС с ИР и без ИР. У пациентов с МС при отсутствии нарушений глюкозо-инсулинового гомеостаза и у пациентов с ИР выявлено накопление полиненасыщенных жирных кислот (18:2n6, 18:3n3, 22:4n6) и снижение пула насыщенных кислот (12:0, 14:0, 16:0, 17:0) в плазме крови [43]. Выявлен дефицит в мембранах эритроцитов полиненасыщенных жирных кислот (18:3n3, 20:4n6) с преобладанием насыщенных ЖК (14:0, 18:0). У лиц с МС независимо от состояния углеводного обмена установлено высокое содержание окисленных производных жирных кислот - лейкотриена $\mathrm{B}_{4}$ и 6-кето-простагландина- $\mathrm{F}_{1 a}$ в сыворотке крови. При развитии ИР у пациентов с МС наблюдается увеличение синтеза тромбоксана $\mathrm{A}_{2}$ Таким образом, липидомный анализ жирных кислот и их окисленных производных может явиться значимым инструментом для изучения механизмов развития МС и его диагностики [43].

Гамма-аминомасляная кислота (gamma-aminobutyric acid (GABA)), d-пироглутаминовая кислота (d-pyroglutamic 
acid (PGA)) и N-ацетил-d-триптофан (N-acetyl-dtryptophan (NAT)) относятся к метаболитам, уровень которых изменяется у пациентов с МС без сердечно-сосудистых заболеваний и СД2 и коррелирует с биомаркерами воспаления [56]. Так, уровни GABA и PGA были поВышены у пациентов с МС, по сравнению с контрольной группой, в то время как NAT был снижен на 90\%. Уровень GABA коррелировал с показателями систолического артериального давления, лептином и эндотоксином, а NAT обратно коррелировал с уровнем глюкозы, триглицеридами, C - реактивным белком, toll-подобным рецептором-4, липополисахарид-связывающим белком, хемерином и ретинол-связывающим белком-4. Таким образом, GABA и PGA активируют воспаление при MC, тогда как NAT смягчает этот ответ.

В последние годы активно изучается биомаркерная роль ряда цитокинов при МС. Хроническое воспаление низкой интенсивности является ключевым фактором развития МС [57]. Этот воспалительный ответ опосредуется инфильтрацией иммунных клеток и экспрессией воспалительных цитокиноB. IL6, секретируемый миелоидными клетками, ингибирует накопление макрофагов жировой ткани. Эти наблюдения дают физиологическое понимание сложности IL6-опосредованной регуляции воспаления [58]. Макрофаги жировой ткани являются основными участниками энергетического обмена. Провоспалительные (M1) макрофаги стимулируют стеатоз печени и адипогенез, тогда как противовоспалительные макрофаги (M2) обладают обратным действием [59, 60]. Ограниченный макрофагами дефицит метил-CpG-связывающего домена белка 2 (methyl-CpG-binding protein 2 (MECP2)) вызывал развитие стеатоза печени и ожирения в экспериментальной модели [61]. Накопление в жировой ткани М1 макрофагов опосредуется альфа-1-интегрином. Задержка и активация макрофагов М1 в жировой ткани ингибируют функции адипоцитов. Роль интегрина альфа-1 в ожирении особенно важна, что подтверждается тем фактом, что FDA одобрила препарат Натализумаб (Natalizumab), который блокирует этот интегрин.

Активно изучается роль некоторых цитокинов, продуцируемых адипоцитами, в качестве биомаркеров МС. Высокая значимость в патофизиологии системного воспаления при ожирении отводится изменению уровней адипокинов - лептина и адипонектина [58]. Являясь основным медиатором воспаления при ожирении, лептин обладает провоспалительным действием [62] и подавляет аппетит, а его генетический дефект вызывает ожирение [63]. Адипонектин повышает чувствительность к инсулину, а также выживаемость и функциональность бета-клеток поджелудочной железы. Гиперэкспрессия адипонектина оказывает положительное влияние на жировую ткань, в частности на увеличение плотности митохондрий, уменьшение размера адипоцитов и транскрипционную активацию факторов, связанных с эстерификацией свободных жирных кислот [64]. В отличие от лептина, экспрессия адипонектина при ожирении снижается, что может быть предиктором развития МС [3].

К биомаркерам МС можно отнести цинк-а 2 -гликопротеин, который является адипокином, участвующим в регуляции липидного обмена [65]. Связь уровня адипонектина и цинк- $a_{2}$-гликопротеина указывает на его роль в развитии ИР и МС. Кроме того, снижение уровня цир- кулирующего цинк-а прогрессирует с увеличением числа его компонентов.

В последнее время костная ткань рассматривается как плейотропный эндокринный орган, секретирующий остеокальцин и липокалин 2 (Lipocalin2 (LCN2)), которые регулируют гомеостаз глюкозы $[66,67]$. Кроме того, LCN2 определяет чувствительность к инсулину, снижает потребление пищи, активируя melanocortin 4 receptor (MC4R)-зависимый путь [68]. Эти результаты идентифицируют LCN2 как гормон, который подавляет аппетит зависимым от MC4R образом, и показывают, что контроль аппетита является эндокринной функцией костной ткани. Возможно, LCN2 можно рассматривать в качестве одного из биомаркеров MC.

Гормоны щитовидной железы являются хорошо известными факторами, регулирующими энергетический обмен $[69,70]$. Недавно было описано взаимодействие гормонов щитовидной железы и рецептора печени (liver x receptor (LXR)) [71]. Снижение экспрессии LXR у мышей приводит к увеличению секреции гормонов щитовидной железы. Кроме того, снижение экспрессии перинатальной дейодиназы 2 (perinatal deiodinase 2) в гепатоцитах уменьшает восприимчивость к развитию стеатоза и ожирения, вызванных высокожировой диетой [71]. Изучение биомаркерной роли гормонов щитовидной железы может быть перспективным для исследований.

Тромбин также способствует развитию ожирения, вызванного высокожировой диетой, через развитие фибринового воспаления. Лечение ингибитором тромбина дабигатраном блокирует развитие ожирения и МС [72]. Добавление витамина D самкам мышей снижает риск развития MC у их потомства [73]. Недавно разработан агонист PPAR, который оказывал положительное влияние на течение МС в экспериментальных условиях [74].

Стресс эндоплазматического ретикулума является еще одним важным фактором в патогенезе MC [75]. В эксперименте на мышах B. Shan и соавт. показали, что стресс, вызванный диетой с высоким содержанием жиров, зависит от активности фермента, нуждающегося в инозитоле (inositol-requiring enzyme 1 alpha (IRE1alpha)) [76]. Его дефицит предотвращал развитие ожирения, ИР и стеатоза печени, вызванных высокожировой диетой.

В то же время стоит отметить, что на сегодняшний день опубликовано немного статей об идентификации биомаркеров и эндогенных метаболитов МС с использованием метаболомики и липидомики у взрослых, поэтому трудно делать однозначные выводы из имеющихся данных [77]. Необходимо более глубокое изучение патофизиологических механизмов МС, которые связывают с достижениями в области метаболического фенотипирования [78]. МС имеет характерный метаболический профиль, однако существует много дискуссионных вопросов о причинно-следственных взаимоотношениях различных компонентов МС и их патофизиологической интеграции. В связи с этим актуальным является выделение подгрупп пациентов с определенными фенотипами МС, которые ассоциируются с высоким риском кардиоваскулярных осложнений. Каким фенотипом проявится MC, зависит от взаимодействия различных факторов (генетических, внешней среды, образа жизни). В этой связи, сочетание метаболомики с исследованиями геномики, транскриптомики и протеомики улучшит понимание 
патофизиологии конкретного клинического фенотипа. Такой подход может быть важным шагом на пути к персонифицированной медицине, при которой индивидуум рассматривается с учетом влияния генетической составляющей, окружающей среды и образа жизни.

\section{ЗАКЛЮЧЕНИЕ}

Исследования по оценке распространенности МС сообщают о противоречивых результатах из-за различий в применяемых диагностических критериях, однако общепризнано, что распространенность данной патологии увеличивается в развитых и развивающихся странах мира, что сопровождается ростом распространенности СД2, гипертонии, сердечно-сосудистых заболеваний и ожирения.

Распространенность МС зависит от многих факторов, способствующих его развитию, что объясняет сложности диагностики и подходов к профилактике и лечению данной патологии. Возраст, факторы образа жизни, социально-экономический статус играют важную роль в развитии МС. Инсулинорезистентность, дисрегуляция липидного обмена, ожирение и генетическая предрасположенность также являются факторами, влияющими на риск развития и прогрессирования МС. Особенности распределения и дисфункция жировой ткани признаны важными факторами развития ИР, связанной с ожирением, а также риском формирования кардиометаболических заболеваний и МС. В то же время лица, страдающие метаболически здоровым ожирением, также имеют риск развития заболеваний, ассоциированных с ожирением, который выше, чем у лиц с нормальным ИМТ. В этой связи детальное метаболическое фенотипирование может служить способом выявления лиц, подверженных повышенному риску развития метаболических заболеваний.
Несмотря на идентификацию многих биомаркеров и эндогенных метаболитов MC, на сегодняшний день опубликовано немного литературных данных об их идентификации с использованием метаболомики и липидомики. Изучение механизмов МС в значительной степени связывают с достижениями в области метаболического фенотипирования, поэтому метаболомика может быть стратегией выбора для глубокого исследования МС и его компонентов.

\section{ДОПОЛНИТЕЛЬНАЯ ИНФОРМАЦИЯ}

Источники финансирования. Исследование проводилось за счет средств федерального бюджета в рамках государственного задания ФНИ. Тема НИР «Изучить клинико-функциональные и метаболические взаимосвязи в рамках метаболического синдрома и разработать пути оптимизации патогенетической терапии», № госрегистрации 01200706095.

Конфликт интересов. Авторы декларируют отсутствие явных и потенциальных конфликтов интересов, связанных с содержанием настоящей статьи.

Участие авторов. Кытикова О.Ю. - концепция и дизайн исследования, подготовка и написание текста статьи; Антонюк М.В. - концепция и дизайн исследования, подготовка текста статьи; Кантур Т.А. анализ данных, подготовка текста статьи, внесение в рукопись существенной правки с целью повышения научной ценности статьи; Новгородцева Т.П. - интерпретация результатов, подготовка текста статьи, внесение принципиальных изменений; Денисенко Ю.К. анализ данных, внесение в рукопись существенной правки с целью повышения научной ценности статьи. Все авторы одобрили финальную версию статьи перед публикацией, выразили согласие нести ответственность за все аспекты работы, подразумевающую надлежащее изучение и решение вопросов, связанных с точностью или добросовестностью любой части работы.

\section{СПИСОК ЛИТЕРАТУРЫ | REFERENCES}

1. Рекомендачии по ведению больных с метаболическим синдромом. Клинические рекомендачии. - М.: 2013. - 43 с. [Rekomendacii po vedeniju bol'nyh s metabolicheskim sindromom. Klinicheskie rekomendacii. Moscow: 2013.43 p. (In Russ.)].

2. Kuschnir MCC, Bloch KV, Szklo M, et al. ERICA: prevalence of metabolic syndrome in Brazilian adolescents. Revista de saúde Pública. 2016;50(11):256-268. doi: https://doi.org/10.1590/S01518-8787.2016050006701

3. Xiang Y, Zhou W, Duan X, et al. Metabolic Syndrome, and Particularly the Hypertriglyceridemic-Waist Phenotype, Increases Breast Cancer Risk, and Adiponectin Is a Potential Mechanism: A Case-Control Study in Chinese Women. Front Endocrinol (Lausanne). 2020;10:905. doi: https://doi.org/10.3389/fendo.2019.00905

4. Lee J, Lee KS, Kim H, et al. The relationship between metabolic syndrome and the incidence of colorectal cancer. Environ Health Prev Med. 2020;25(1):6. doi: https://doi.org/10.1186/s12199-020-00845-w

5. Grgurevic I, Podrug K, Mikolasevic I, et al. Natural History of Nonalcoholic Fatty Liver Disease: Implications for Clinical Practice and an Individualized Approach. Can J Gastroenterol Hepatol. 2020;2020:1-10. doi: https://doi.org/10.1155/2020/9181368

6. Wang Y, Tu R, Yuan $\mathrm{H}$, et al. Associations of unhealthy lifestyles with metabolic syndrome in Chinese rural aged females. Sci Rep. 2020;10(1):2718. doi: https://doi.org/10.1038/s41598-020-59607-x

7. Lee M-K, Han K, Kim MK, et al. Changes in metabolic syndrome and its components and the risk of type 2 diabetes: a nationwide cohort study. Sci Rep. 2020;10(1):2313. doi: https://doi.org/10.1038/s41598-020-59203-z

8. Lee M-K, Han K, Kim MK, et al. Health Effects of Overweight and Obesity in 195 Countries over 25 Years. N Engl J Med. 2017;377(1):13-27. doi: https://doi.org/10.1056/NEJMoa1614362
9. Brandão AD, da Silva JH, Mariane Oliveira Lima S, et al. Short and long term effect of treatment non-pharmacological and lifestyle in patients with metabolic syndrome. Diabetol Metab Syndr. 2020;12(1):16. doi: https://doi.org/10.1186/s13098-020-0522-y

10. Bloomgarden ZT. Symposium: Debating the Metabolic Syndrome. Medscape Conference Coverage, based on selected sessions. American Diabetes Association 66th Scientific Sessions. Washington: DC; 2006; June 9-13.

11. Grundy SM, Cleeman Jl, Daniels SR, et al. Diagnosis and Management of the Metabolic Syndrome. Circulation. 2005;112(17):35-52. doi: https://doi.org/10.1161/CIRCULATIONAHA.105.169405

12. Ogurtsova $K$, da Rocha Fernandes JD, Huang Y, et al. IDF Diabetes Atlas: Global estimates for the prevalence of diabetes for 2015 and 2040. Diabetes Res Clin Pract. 2017;128:40-50. doi: https://doi.org/10.1016/j.diabres.2017.03.024

13. Ranasinghe $P$, Mathangasinghe $Y$, Jayawardena $R$, et al. Prevalence and trends of metabolic syndrome among adults in the asiapacific region: a systematic review. BMC public. health. 2017;17:101. doi: https://doi.org/10.1186/s12889-017-4041-1

14. Антонюк М.В., Новгородцева Т.П., Денисенко Ю.К., и др. Метаболический синдром. Актуальные вопросы диагностики, патогенеза и восстановительного лечения: монография. Владивосток: Изд- во Дальневост. федерал. ун-та; 2018. - 212 с. [Antonjuk MV, Novgorodceva TP, Denisenko JuK, et al. Metabolicheskij sindrom. Aktual'nye voprosy diagnostiki, patogeneza i vosstanovitel'nogo lechenija: monografija. Vladivostok: Izd-vo Dal'nevost. federal. un-ta; 2018. 212 p. (In Russ.)].

15. Alberti KGMM, Eckel RH, Grundy SM, et al. Harmonizing the Metabolic Syndrome. Circulation. 2009;120(16):1640-1645. doi: https://doi.org/10.1161/CIRCULATIONAHA.109.192644 
16. Expert Panel on Detection, Evaluation and T of HBC in A. Executive Summary of the Third Report of the National Cholesterol Education Program (NCEP) Expert Panel on Detection, Evaluation, and Treatment of High Blood Cholesterol in Adults (Adult Treatment Panel III). JAMA J Am Med Assoc. 2001;285(19):2486-2497. doi: https://doi.org/10.1001/jama.285.19.2486

17. Bloomgarden ZT. American Association of Clinical Endocrinologists (AACE) Consensus Conference on the Insulin Resistance Syndrome: 25-26 August 2002, Washington, DC. Diabetes Care. 2003;26(4):1297-1303. doi: https://doi.org/10.2337/diacare.26.4.1297

18. Wu LT, Shen YF, Hu L, et al. Prevalence and associated factors of metabolic syndrome in adults: a population-based epidemiological survey in Jiangxi province, China. BMC Public Health. 2020;20(1):133. doi: https://doi.org/10.1186/s12889-020-8207-x

19. Moore JX, Chaudhary N, Akinyemiju T. Metabolic Syndrome Prevalence by Race/Ethnicity and Sex in the United States, National Health and Nutrition Examination Survey, 1988-2012. Prev Chronic Dis. 2017;14:160287. doi: https://doi.org/10.5888/pcd14.160287

20. Ferguson-Smith AC, Patti ME. You are what your dad ate. Cell Metab. 2011;13(2):115-117 doi: https://doi.org/10.1016/j.cmet.2011.01.011

21. Hardy DS, Garvin JT, Mersha TB, Racette SB. Ancestry specific associations of FTO gene variant and metabolic syndrome. Medicine (Baltimore). 2020;99(6):e18820. doi: https://doi.org/10.1097/MD.0000000000018820

22. Lee H-S, Kim Y, Park T. New Common and Rare Variants Influencing Metabolic Syndrome and Its Individual Components in a Korean Population. Sci Rep. 2018;8(1):5701. doi: https://doi.org/10.1038/s41598-018-23074-2

23. Musani SK, Martin LJ, Woo JG, et al. Heritability of the Severity of the Metabolic Syndrome in Whites and Blacks in 3 Large Cohorts. Circ Cardiovasc Genet. 2017;10(2) doi: https://doi.org/10.1161/CIRCGENETICS.116.001621

24. Sigit FS, Tahapary DL, Trompet S, et al. The prevalence of metabolic syndrome and its association with body fat distribution in middleaged individuals from Indonesia and the Netherlands: a crosssectional analysis of two population-based studies. Diabetol Metab Syndr. 2020;12(1):2. doi: https://doi.org/10.1186/s13098-019-0503-1

25. Palaniappan LP, Wong EC, Shin JJ. et al. Asian Americans have greater prevalence of metabolic syndrome despite lower body mass index. In J Obe. 2017;35:393-400. doi: https://doi.org/10.1038/ijo.2010.152.

26. Huang $X$, Hu Y, Du L, et al. Metabolic syndrome in native populations living at high altitude: a cross-sectional survey in Derong, China. BMJ Open. 2020;10(1):e032840. doi: https://doi.org/10.1136/bmjopen-2019-032840

27. Ivanova N, Liu Q, Agca C, et al. White matter inflammation and cognitive function in a co-morbid metabolic syndrome and prodromal Alzheimer's disease rat model. J Neuroinflammation. 2020;17(1):29. doi: https://doi.org/10.1186/s12974-020-1698-7

28. Nolan PB, Carrick-Ranson G, Stinear JW. et al. Prevalence of metabolic syndrome and metabolic syndrome components in young adults: A pooled analysis. Prev. Med. Rep. 2017;7:211-215. doi: https://doi.org/10.1016/j.pmedr.2017.07.004

29. Mozaffarian D, Benjamin EJ, Go AS. et al. Heart disease and stroke statistics--2015 update: a report from the American Heart Association. Circulation. 2015;131:e29-322. doi: https://doi.org/10.1161/CIR.0000000000000152.

30. Liu J-H, Qian Y-X, Ma Q-H, et al. Depressive symptoms and metabolic syndrome components among older Chinese adults. Diabetol Metab Syndr. 2020;12(1):18. doi: https://doi.org/10.1186/s13098-020-00526-2

31. Fan L, Hao Z, Gao L, et al. Non-linear relationship between sleep duration and metabolic syndrome. Medicine (Baltimore). 2020;99(2):e18753. doi: https://doi.org/10.1097/MD.0000000000018753

32. Selvi Y, Kandeger A, Boysan M. et al. The effects of individual biological rhythm differences on sleep quality, daytime sleepiness, and dissociative experiences. Psychiatry Res. 2017;256:243-248. doi: https://doi.org/10.1016/j.psychres.2017.06.059

33. 1. Liao C-C, Sheu WH-H, Lin S-Y, et al. The Relationship Between Abdominal Body Composition and Metabolic Syndrome After a Weight Reduction Program in Adult Men with Obesity. Diabetes, Metab Syndr Obes Targets Ther. 2020;13:1-8. doi: https://doi.org/10.2147/DMSO.S228954
34. Goh VHH, Hart WG. Excess fat in the abdomen but not general obesity is associated with poorer metabolic and cardiovascular health in premenopausal and postmenopausal Asian women. Maturitas. 2018;107:33-38 doi: https://doi.org/10.1016/j.maturitas.2017.10.002

35. Ladeiras-Lopes R, Sampaio F, Bettencourt N., et al. The ratio between visceral and subcutaneous abdominal fat assessed by computed tomography is an independent predictor of mortality and cardiac events. Rev Esp Cardiol (Engl Ed). 2017;70(5):331-337. doi: https://doi.org/10.1016/j.recesp.2016.09.006

36. Филатова Г.А., Дэпюи Т.И., Гришина Т.И. Ожирение: спорные вопросы, определяющие метаболическое здоровье // Эндокринология: новости, мнения, обучение. - 2018. - Т. 7. №1. - C. 58-67. [Filatova GA, Djepjui TI, Grishina TI. Ozhirenie: spornye voprosy, opredeljajushhie metabolicheskoe zdorov'e. Jendokrinologija: novosti, mnenija, obuchenie. 2018;7(1):58-67. (In Russ.)].

37. van Vliet-Ostaptchouk JV, Nuotio M-L, Slagter SN, et al. The prevalence of metabolic syndrome and metabolically healthy obesity in Europe: a collaborative analysis of ten large cohort studies. BMC Endocr Disord. 2014;14(1):9. doi: https://doi.org/10.1186/1472-6823-14-9

38. Phillips CM. Metabolically healthy obesity across the life course: epidemiology determinants, and implications. Ann N Y Acad Sci. 2017;1391(1):85-100. doi: https://doi.org/1111/nyas.13230

39. Brown RE, Kuk JL. Consequences of obesity and weight loss: a devil's advocate position. Obes. Rev. 2015;16(1):77-87. doi: https://doi.org/10.1111/obr.12232

40. Eckel N, Meidtner K, Kalle-Uhlmann T, et al. Metabolically healthy obesity and cardiovascular events: a systematic review and meta-analysis. Eur. J. Prev. Cardiol. 2016;23(9):956-966. doi: https://doi.org/10.1177/2047487315623884

41. Chen TP, Lin WY, Chiang CH, et al. Metabolically healthy obesity and risk of non-alcoholic fatty liver disease severity independent of visceral fat. J Gastroenterol Hepatol. 2021. doi: 10.1111/jgh.15544.

42. Kytikova OY, Antonyuk MV, Gvozdenko TA, Novgorodtseva TP. Metabolic aspects of the relationship of asthma and obesity. Obesity and Metabolism. 2019;15(4):9-14. doi: https://doi.org/10.14341/omet9578

43. Novgorodtseva TP, Karaman YK, Zhukova NV, et al. Composition of fatty acids in plasma and erythrocytes and eicosanoids level in patients with metabolic syndrome. Lipids Health Dis. 2011;10(1):82. doi: https://doi.org/10.1186/1476-511X-10-82

44. Niu H, Zhang H, Peng J, et al. Quantitative endogenous peptidomics analysis of the type-2 diabetic clinical serum samples. Chinese J Chromatogr. 2019;37(8):853. doi: https://doi.org/10.3724/SP.J.1123.2019.03012

45. Chen D, Zhao X, Sui Z, et al. A multi-omics investigation of the molecular characteristics and classification of six metabolic syndrome relevant diseases. Theranostics. 2020;10(5):2029-2046. doi: https://doi.org/10.7150/thno.41106

46. Surowiec I, Noordam R, Bennett K, et al. Metabolomic and lipidomic assessment of the metabolic syndrome in Dutch middle-aged individuals reveals novel biological signatures separating health and disease. Metabolomics. 2019;15(2):23. doi: https://doi.org/10.1007/s11306-019-1484-7

47. Liggi S, Griffin JL. Metabolomics applied to diabetes-lessons from human population studies. Int. J. Biochem. Cell Biol. 2017;93:136-147. doi: https://doi.org/10.1016/j.biocel.2017.10.011

48. Gao X, Tian Y, Randell E, et al. Unfavorable Associations Between Serum Trimethylamine N-Oxide and L-Carnitine Levels With Components of Metabolic Syndrome in the Newfoundland Population. Front Endocrinol (Lausanne). 2019;10. doi: https://doi.org/10.3389/fendo.2019.00168

49. Barrea L, Annunziata G, Muscogiuri G, et al. Trimethylamine$\mathrm{N}$-oxide (TMAO) as Novel Potential Biomarker of Early Predictors of Metabolic Syndrome. Nutrients. 2018;10(12):1971. doi: https://doi.org/10.3390/nu10121971

50. Plovier $\mathrm{H}$, Everard A, Druart $\mathrm{C}$, et al. A purified membrane protein from Akkermansia muciniphila or the pasteurized bacterium improves metabolism in obese and diabetic mice. Nat Med. 2017;23(1):107-113. doi: https://doi.org/10.1038/nm.4236

51. Zeng S-L, Li S-Z, Xiao P-T, et al. Citrus polymethoxyflavones attenuate metabolic syndrome by regulating gut microbiome and amino acid metabolism. Sci Adv. 2020;6(1):6208. doi: https://doi.org/10.1126/sciadv.aax6208 
52. Ouchi S, Shimada K, Miyazaki T, et al. Low 1,5-anhydroglucitol levels are associated with long-term cardiac mortality in acute coronary syndrome patients with hemoglobin A1C levels less than 7.0\%. Cardiovasc Diabetol. 2017;16(1):151. doi: https://doi.org/10.1186/s12933-017-0636-1

53. Zhenyukh O, Civantos E, Ruiz-Ortega M, et al. High concentration of branched-chain amino acids promotes oxidative stress, inflammation and migration of human peripheral blood mononuclear cells via mTORC1 activation. Free Radic Biol Med. 2017;104:165-177 doi: https://doi.org/10.1016/j.freeradbiomed.2017.01.009

54. Aichler M, Borgmann D, Krumsiek J, et al. N-acyl Taurines and Acylcarnitines Cause an Imbalance in Insulin Synthesis and Secretion Provoking $\beta$ Cell Dysfunction in Type 2 Diabetes. Cell Metab. 2017:25(6):1334-1347. doi: https://doi.org/10.1016/j.cmet.2017.04.012

55. Ramakrishanan N, Denna T, Devaraj S, et al. Exploratory lipidomics in patients with nascent Metabolic Syndrome. J Diabetes Complications. 2018;32(8):791-794. doi: https://doi.org/10.1016/j.jdiacomp.2018.05.014

56. Shim K, Gulhar R, Jialal I. Exploratory metabolomics of nascent metabolic syndrome. J Diabetes Complications. 2019;33(3):212-216. doi: https://doi.org/10.1016/j.jdiacomp.2018.12.002

57. Grandl G, Wolfrum C. Hemostasis, endothelial stress, inflammation, and the metabolic syndrome. Semin Immunopathol. 2018;40(2):215-224. doi: https://doi.org/10.1007/s00281-017-0666-5

58. Han MS, White A, Perry RJ, et al. Regulation of adipose tissue inflammation by interleukin 6. Proc Natl Acad Sci. 2020;117(6):2751-2760. doi: https://doi.org/10.1073/pnas.1920004117

59. Mouton AJ, Li X, Hall ME, et al. Obesity, Hypertension, and Cardiac Dysfunction: Novel Roles of Immunometabolism in Macrophage Activation and Inflammation. Circ Res. 2020;126(6):789-806. doi: https://doi.org/10.1161/CIRCRESAHA.119.312321

60. Dini L, Tacconi S, Carata E, et al. Microvesicles and exosomes in metabolic diseases and inflammation. Cytokine Growth Factor Rev. 2020;51:27-39. doi: https://doi.org/10.1016/j.cytogfr.2019.12.008

61. Jing Y, Wu F, Li D, et al. Metformin improves obesityassociated inflammation by altering macrophages polarization. Mol Cell Endocrinol. 2018;461:256-264. doi: https://doi.org/10.1016/.mce.2017.09.025

62. Khodamoradi K, Parmar M, Khosravizadeh Z, et al. The role of leptin and obesity on male infertility. Curr Opin Urol. 2020;30(3):334-339. doi: https://doi.org/10.1097/MOU.0000000000000762

63. Shiba CK, Dâmaso AR, Rhein SO, et al. Interdisciplinary therapy had positive effects on inflammatory state, mediated by leptin adiponectin, and quality of diet in obese women. Nutr Hosp. 2020 doi: https://doi.org/10.20960/nh.02777

64. Benrick A, Chanclón B, Micallef $\mathrm{P}$, et al. Adiponectin protects against development of metabolic disturbances in a PCOS mouse model. Proc Natl Acad Sci. 2017;114(34):E7187-E7196. doi: https://doi.org/10.1073/pnas.1708854114

65. Lei L, Li K, Li L, et al. Circulating zinc-a2-glycoprotein levels are low in newly diagnosed patients with metabolic syndrome and correlate with adiponectin. Nutr Metab (Lond). 2017:14(1):53. doi: https://doi.org/10.1186/s12986-017-0210-6

66. Urbschat A, Thiemens A-K, Mertens C, et al. Macrophage-Secreted Lipocalin-2 Promotes Regeneration of Injured Primary Murine Renal Tubular Epithelial Cells. Int J Mol Sci. 2020;21 (6):2038. doi: https://doi.org/10.3390/ijms21062038

67. Currò D, Vergani E, Bruno C, et al. Plasmatic lipocalin-2 levels in chronic low-grade inflammation syndromes: Comparison between metabolic syndrome, total and partial adult growth hormone deficiency. BioFactors. 2020;46(4):629-636. doi: https://doi.org/10.1002/biof.1628

68. Mosialou I, Shikhel S, Liu J-M, et al. MC4R-dependent suppression of appetite by bone-derived lipocalin 2. Nature. 2017:543(7645):385-390. doi: https://doi.org/10.1038/nature21697

69. Nie X, Ma X, XuY, et al. Characteristics of Serum Thyroid Hormones in Different Metabolic Phenotypes of Obesity. Front Endocrinol (Lausanne). 2020;11:68. doi: https://doi.org/10.3389/fendo.2020.00068

70. Amouzegar A, Kazemian E, Abdi H, et al. Association Between Thyroid Function and Development of Different Obesity Phenotypes in Euthyroid Adults: A Nine-Year Follow-Up. Thyroid 2018;28(4):458-464. doi: https://doi.org/10.1089/thy.2017.0454

71. Miao Y, Warner M, Gustafsson J-Å. Liver X receptor $\beta$ : new player in the regulatory network of thyroid hormone and 'browning' of white fat. Adipocyte. 2016;5(2):238-242 doi: https://doi.org/10.1080/21623945.2016.1142634

72. Kopec AK, Abrahams SR, Thornton S, et al. Thrombin promotes diet-induced obesity through fibrin-driven inflammation. J Clin Invest. 2017;127(8):3152-3166. doi: https://doi.org/10.1172/JC192744

73. Villa CR, Chen J, Wen B, et al. Maternal vitamin D beneficially programs metabolic, gut and bone health of mouse male offspring in an obesogenic environment. In J obes. 2016;40(12):1875-1883. doi: 10.1038/ijo.2016.177.

74. Cox RL. Rationally designed PPARdelta-specific agonists and therapeutic potential for metabolic syndrome. Proc Natl Acad Sci USA. 2017;114:3284-3285 doi: https://doi.org/10.1073/pnas.1702084114.

75. Alicka M, Marycz K. The Effect of Chronic Inflammation and Oxidative and Endoplasmic Reticulum Stress in the Course of Metabolic Syndrome and Its Therapy. Stem Cells Int. 2018;2018:1-13. doi: https://doi.org/10.1155/2018/4274361

76. Shan B, Wang X, Wu Y, et al. The metabolic ER stress sensor IRE1 alpha suppresses alternative activation of macrophages and impairs energy expenditure in obesity. Nat immunology. 2017;18(5):519-529. doi: https://doi.org/10.1038/ni.3709

77. Monnerie S, Comte B, Ziegler D, et al. Metabolomic and Lipidomic Signatures of Metabolic Syndrome and its Physiological Components in Adults: A Systematic Review. Sci Rep. 2020;10(1):669. doi: https://doi.org/10.1038/s41598-019-56909-7

78. Dumas M, Kinross J, Nicholson JK. Metabolic Phenotyping and Systems Biology Approaches to Understanding Metabolic Syndrome and Fatty Liver Disease. Gastroenterology 2014;146(1):46-62. doi: https://doi.org/10.1053/j.gastro.2013.11.001

\section{ИНФОРМАЦИЯ ОБ АВТОРАХ [AUTHORS INFO]:}

Кытикова Оксана Юрьевна, д.м.н. [Охаna Yu. Kytikova, MD, PhD]; адрес: Россия, 690105, Владивосток, ул. Русская, д. 73 Г [address: 73 G Russkaya street, 690105, Vladivostok, Russia]; ORCID: https://orcid.org/0000-0001-5018-0271; eLibrary SPIN: 3006-5614; e-mail: kytikova@yandex.ru

Антонюк Марина Владимировна, д.М.н., професcop [Marina V. Antonyuk, MD, PhD, Professor]; ORCID: https://orcid.org/0000-0002-2492-3198; eLibrary SPIN: 3446-4852; e-mail: antonyukm@mail.ru

Кантур Татьяна Александровна, к.м.н. [Tatyana A. Kantur, MD, PhD]; ORCID: https://orcid.org/0000-0002-7851-6141; eLibrary SPIN: 7889-7290; e-mail: kanturovichi@yandex.ru

Новгородцева Татьяна Павловна, д.б.н., професcop [Tatyana P. Novgorodtseva, MD, PhD, Professor]; ORCID: https://orcid.org/0000-0002-6058-201X; eLibrary SPIN: 5888-6099; e-mail: nauka@niivl.ru Денисенко Юлия Константиновна, д.б.н. [Yuliya K. Denisenko, MD, PhD]; ORCID: https://orcid.org/0000-0003-4130-8899; eLibrary SPIN-код: 4997-3432; e-mail: karaman@inbox.ru

*Автор, ответственный за переписку / Corresponding author. 


\section{ЦИТИРОВАТЬ:}

Кытикова О.Ю., Антонюк М.В., Кантур Т.А., Новгородцева Т.П., Денисенко Ю.К. Распространенность и биомаркеры метаболического синдрома // Ожирение и метаболизм. — 2021. — Т. 18. — №3. — С. $302-312$. doi: https://doi.org/10.14341/omet12704

\section{TO CITE THIS ARTICLE:}

Kytikova OY, Antonyuk MV, Kantur TA, Novgorodtseva TP, Denisenko YK. Prevalence and biomarkers in metabolic syndrome. Obesity and metabolism. 2021;18(3):302-312. doi: https://doi.org/10.14341/omet12704 